Published in "Journal of ambient intelligence and smart environments", 2016, vol. 8, no 2, pp. 219-237, which should be cited to refer to this work.

\title{
An Expert Personal Health System to Monitor Patients Affected by Gestational Diabetes Mellitus: A Feasibility Study
}

\author{
Bromuri Stefano ${ }^{\mathrm{a}, *}$, Puricel Serban ${ }^{\mathrm{b}}$ and Schumann Rene ${ }^{\mathrm{a}}$ and Krampf Johannes ${ }^{\mathrm{a}}$ and Ruiz Juan ${ }^{\mathrm{c}}$ and \\ Schumacher Michael ${ }^{\mathrm{a}}$ \\ ${ }^{\text {a }}$ University of Applied Sciences Western Switzerland, Institute of Business Information Systems, Sierre, \\ Switzerland. \\ ${ }^{\mathrm{b}}$ Department of Cardiology, University of Fribourg, Fribourg, Switzerland. \\ ${ }^{\mathrm{c}}$ Hospital Riviera-Chablais, Bd Paderewski 3, 1800 Vevey, Switzerland.
}

\begin{abstract}
.
Purpose: Gestational Diabetes Mellitus (GDM) is a condition affecting 3-4\% of pregnant women due to increased resistance to insulin caused by the growth of the fetus. Such a condition disappears just after delivery, but it is an indicator of the insurgence of diabetes type 2 (DT2) later in life: about $40 \%$ of the women affected by GDM also develop DT2 [22]. GDM brings several complications during pregnancy to both the mother and the fetus. We aim here at presenting our Personal Health System for monitoring GDM and we also present the results of outpatient monitoring and management by utilizing a personal health system (PHS) for GDM.

Methods: The Personal Health System (PHS) was deployed in a feasibility study, modelled as a single-center, parallel group, open randomized controlled trial conducted in Lausanne University Hospital. Patients $(n=24)$ were assigned to 2 different groups: standard protocol group (SP) and telemedicine group (TM). SP patients were managed by regular clinic visits. TM patients were managed with our EPHS system. The targeted feasibility outcome was whole trial feasibility, functioning of the PHS and its appropriateness for patient use.

Results: Mean age was $32 \pm 5$ years and patients were pregnant for $29.1 \pm 1.9$ weeks at study inclusion. Patients came from 16 different countries. The follow-up rate was $100 \%$. Acceptability in the TM-group was high, as $100 \%$ were satisfied with the care provided and equally $100 \%$ were at ease with the technology. Overall median[IQR] glucose control was 5.4 mmol/1 [4.7-6.4] in the TM-group and 5.7mmol/1 [4.9-6.7] in the SP-group ( $\mathrm{p}<0.001)$. Four out of 6 daily plasma glucose values were significantly better controlled with telemedicine compared to standard care.

Conclusion: The feasibility study that we conducted shows that PHSs have a great potential to improve the life of the patient by allowing a better communication of their physiological values to the caregivers. With respect to the particular case of GDM, the study suggests that use of PHS technology may improve glycaemic control in GDM, but to confirm this trend, a main trial is needed.
\end{abstract}

Keywords:

Personal Health Systems, Gestational Diabetes Mellitus, Diabetes, Expert Systems, Agents, Telemedicine.

${ }^{*}$ Corresponding author. E-mail: stefano.bromuri@hevs.ch.

\section{Introduction}

Gestational Diabetes Mellitus (GDM) is a condition affecting $3-4 \%$ of pregnant women due to increased resistance to insulin caused by the growth of the baby. 
Such a condition disappears just after delivery, but it is an indicator of the insurgence of diabetes type 2 (DT2) later in life: about $40 \%$ of the women affected by GDM also develop DT2 [22]. A GDM patient with poor glucose control has also the tendency to develop additional conditions, such as preeclampsia [30], an hypertensive state in which the patient has protenuria in the urine, a condition that may lead to a coma, or macrosomia [39,6], a situation where the fetus grows too much.

The risks for the baby include the following conditions [33,42]: being large for gestational age (macrosomia) causing delivery problems; low blood sugar (hypoglycaemia) after birth due to too high insulin production; yellow skin (jaundice); respiratory distress syndrome; fetal death; childhood obesity.

In normal pregnancies the rate of macrosomia occurrence is about $10 \%$, while in pregnancies where GDM is involved, this rate is two or three times higher [40]. This rate drops to $14 \%$ to $18 \%$ with nutrition counseling. The main risks of macrosomia for the new baby are shoulder dystocia, clavical fractures, and brachial plexus injury forcing in many cases a cesarean section.

Risk factors to develop gestational diabetes include [33]: previous GDM; first degree relative with type 2 diabetes; advanced maternal age; ethnicity; overweight or obesity; previous macrosomia.

According to the current medical practice [33], for a GDM patient the periods and the targets for checking glucose are defined as: a) fasting, a glucose sample taken before breakfast whose value should be between 4 to $5.3 \mathrm{mmol} / \mathrm{l}$ to be within the targets specified in the guidelines; b) postprandial breakfast, a glucose sample taken 2 hours after breakfast, whose value should be be between 4 to $7 \mathrm{mmol} / \mathrm{l}$; c) preprandial lunch, a glucose sample taken before lunch, whose value should be between 4 to $5.3 \mathrm{mmol} / \mathrm{l}$; d) postprandial lunch, a glucose sample taken 2 hours after lunch, whose value should be between 4 to $7 \mathrm{mmol} / \mathrm{l}$; e) preprandial dinner, a glucose sample taken before dinner, its value should be between 4 to $5.3 \mathrm{mmol} / \mathrm{l}$; f) postprandial dinner, a glucose sample taken 2 hours after dinner, its value should be between 4 to $7 \mathrm{mmol} / \mathrm{l}$.

Currently, patients visit the doctor weekly or once every two weeks. This implies that if the patient develops a bad glycaemic control, then the baby may develop macrosomia before the medical doctor in charge of the patient can take any action. In this paper, we present a solution to monitor patients affected by GDM using a Personal Health System (PHS). PHSs are system designed to close the loop between the patient and the medical doctor, to realize the vision of pervasive healthcare [38], which aims at bringing healthcare everywhere and to everyone. The objective of this work is to study the introduction of PHS in the care of patients affected by GDM, to understand whether this approach can be comparable to a standard treatment in terms of glucose control and improve the reaction of the medical staff concerning situations that require further attention. Additionally, we are motivated by the research question of modelling medical knowledge in PHSs, which we try to answer, in this contribution, by means of logic programming and temporal rules in terms of the Event Calculus (EC) [23].

In the research landscape several solutions have been defined to monitor chronically ill patients [29], activity monitoring [37], depressed patients monitoring [34], chronic heart failure [18], and diabetes [36]. In this research an important fact has emerged: despite the usefulness of reporting on all the data of the patient, interconnectivity and telemedicine solutions have only a limited impact in improving the delivery of care as the medical doctor is overwhelmed by the amount of unprocessed information, and, as it emerges in a recent review [16] of PHSs, the satisfaction of the end user is of importance towards the adoption of the technology. Very often an endocrinologist is in charge of many patients at once. Consequently, recent PHS solutions have rather focused on presenting events of interest to the medical doctors, to make the PHS more time effective.

In particular, it is becoming of importance to be able to enact the known medical practice concerning an illness when an issue presents and not to wait until the next time the patient meets the doctor. Enacting the medical practice implies modeling the existing medical knowledge within an expert system, in a way that events of interest get notified to the medical doctors in charge of the patient.

From the perspective of medical knowledge management, logic programming has previously been employed with success for this task, see [19], [28] for two examples.

To model the reasoning part of our PHS, we decided to use an agent based approach. Agents are programmed as autonomous software entities that pursue a set of goals [41] by applying AI reasoning techniques such as logic based reasoning. We found that modeling each agent as a GDM expert system associated to a patient simplified the definition of the architecture of our system, allowing us to separate reasoning components from data components. 
This paper proposes two main contributions: a technical contribution, showing an application of an expert system, and a medical one.

The technical contribution of this paper is to present an application of an expert system, modeled as a complete PHS solution based on expert agents to monitor patients affected by GDM. In this solution, the existing medical knowledge is modeled using a logic programming approach to provide alerts about the glycaemic values of the patients to medical doctors and caregivers. This is a novel contribution as current PHS are only starting now to use sophisticate approaches to deal with the temporal patterns in the physiological values of the patient, and in so far the main focus was providing interconnectivity rather than data analysis and automatic reporting of issues.

Incomplete parts of the current system were published in previous conferences [7,8,24,32]. With respect to such publications, here we present the full final version of our system.

The medical contribution of this paper is to present results of a feasibility study of our PHS carried out at the University Hospital of Lausanne. The feasibility study took the form of a randomized controlled trial where 12 patients were enrolled in the telemedicine arm (TM) and 12 patients were given the standard treatment, the patients were monitored in the time lapse of 9 months using our PHS. This is significant because the current trials have focused on studying PHSs that would rather deal with the simple submission of the values to a database for later consultation, whereas in our case we consider also the reporting of alerts to the medical doctors, by following a medical knowledge engineering approach where the rules for the reporting are modelled according to the practice of the medical doctors and nurses dealing with the GDM patients.

From a methodological perspective, this approach was selected because, given the same population on which the sample of patients is taken, it allows a comparison between standard care and expert based telemedicine care. In this sense, this was important to understand if the two approaches are comparable from a perspective of glucose control and whether the telemedicine system can effectively improve the reaction of the medical doctors, by at the same time being comfortable for the patients.

The evaluation proposed is both quantitative and qualitative as we report on: glucose control of control and telemedicine group, usage of the system from the patient perspective, usage of the system from the doctor perspective, patient's compliance to the treatment in terms of reported glycaemic values, precision of the hyperglycaemia detection rules. In more details, the main information that we wanted to obtain out of the conducted feasibility study was to understand if it is feasible to use the presented PHS in place or in addition of the current standard treatment. From this perspective, the glucose control and the accuracy of the alerts produced by our PHS is important to understand if the alerts produced by our telemedicine system would hinder the glucose control obtained with the standard treatment or they would allow to keep a similar performance. Secondly, the amount of measurements obtained and the reaction time are important to evaluate the usability of our PHS. Thirdly, the opinion of patients and doctors was also considered to evaluate the acceptability of the approach.

Being a feasibility study, it is important to state that the sample of patients considered for such a study does not allow to draw final conclusions on weather the system can improve glucose control of the patients and that a bigger trial is needed for this purpose. Nevertheless, the results obtained are encouraging and can provide some important insights to improve the definition of Expert based PHSs as one result that we can observe with our system is that the glucose control seems to be comparable, the quality and quantity of the data improves and both patients and doctors see benefits in using the technology.

The rest of the paper is structured as follows: Section 2 presents our system in details; Section 3 presents the evaluation of our PHS; Section 4 presents relevant related work; finally Section 5 concludes this work and proposes directions for future work.

\section{Materials and Methods}

Figure 1 shows the general architecture of our system. For our system we consider different types of clients: Web-browsers and an Android application. We use a REST approach based on a HTTPS Security Proxy for server communication. The REST server acts as a single point of entry.

In the Business Layer Module we serve requests, and control the data flow. The Data Access Layer manages the data to be stored in the system database.

We define agents using the multi-agent GOLEM platform [10]. In our system an agent is responsible for monitoring the condition of one patient. To scale-up the reasoning, the agents can be distributed to differ- 
ent nodes, activated and deactivated by a balancer, or persisted in an agent database.

The GOLEM balancer is responsible for handling the incoming requests to the GOLEM agent environment (GE) by splitting the load amongst the GOLEM containers registered with the GOLEM balancer. In particular, when a new request is produced, the GOLEM balancer selects the container that has fewer active agents to put in execution a new agent for patient's monitoring purposes. The details on the scalability of GOLEM were previously presented in [9].

In the remainder of this Section we will go into the details of the data model used, the Android application on the patient's side, the reasoning performed by our agent based system, and the Web application on the caregivers' side.

\subsection{The Data Model}

Within our data model we take into consideration the actors of the system, such as medical caretakers and patients, the treatments and the physiological values of the patient. We follow an object oriented approach where nurses, dietitians and doctors entities are specializations of a generic medical caretaker entity. In our data model, patients are complex entities that can have multiple observations. In particular, for the medical doctors that conducted the experimentation, the following data was of importance to properly characterise GDM: patient's weight, starting date and delivery date of the pregnancy, smoking habits, ethnicity, employment, annual income, level of education, allergies, previous GDM occurrence, history of diabetes type 2 in the family, sport habits, current medications, other illnesses, cholesterol, triglyceride and name of the general practitioner.

In addition to these observations, that are usually collected in the hospital in form of lab tests, for the monitoring GDM with our personal health system we consider the following observations: blood sugar, weight, reports on treatments, blood pressure, and symptoms. Relevant symptoms that need to be considered are: epigastric pain, dyspnea, blurred vision, chest pain, headache, and oedema. In our data model observations and symptoms can have a time duration specified as a time window.

GDM has a number of possible treatments that we model in our system in terms of doses of a drug taken at a certain point in time. We model the following treatments: Novorapid (a fast insulin), Humalog (a fast in- sulin), Insulatard (a slow insulin), Huminsulinbasale (a slow insulin) and Metformin.

The periods of the day when the treatment can be taken are then: in the morning, at lunch, in the afternoon, in the evening, in the night.

\subsection{The Patient's Android Interface}

In order to provide physiological values to our system, patients are given a mobile phone with an application to enter blood sugar measurements, medicine taken by the patient and any symptoms related to GDM. Furthermore, patients can visualize their blood sugar measurements and symptoms, look at the entered vales in a tabular format and correct mistyped values.

To access the application, we use a double mean of authentication. As a first mean of authentication, we store an encrypted certificate within the smart phone in binary format. Then, we provide our patients with a QR code, containing the keys for the certificate store and for encryption on the phone. This is described in Figure 2.

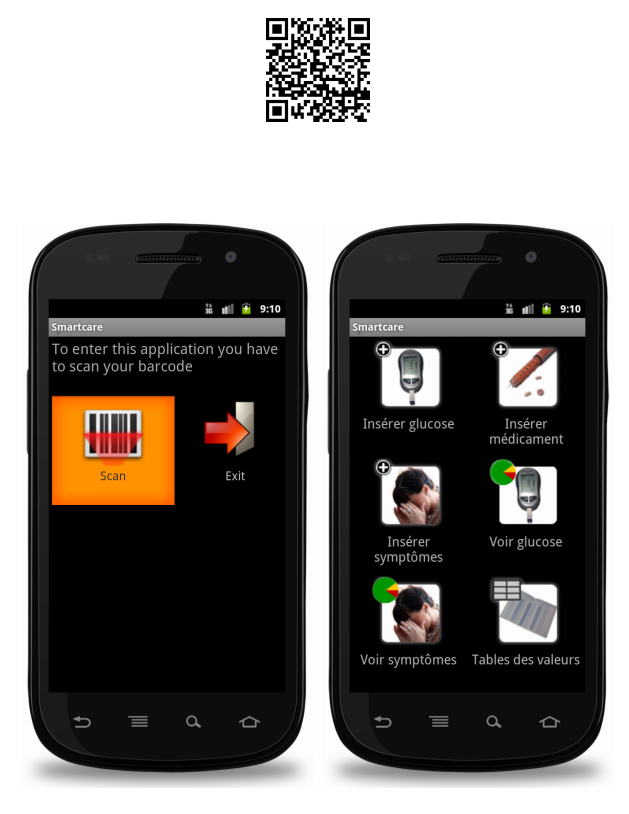

Fig. 2. Security for the Mobile Phone Application Interface.

Consequently, in order to steal the identity of a patient, it is not enough to have the hand-held device, it is also necessary to have the QR code. Furthermore, the protection on the transportation layer is achieved using a mutually authenticated HTTPS connection between the smart phone and backend system implied. The stor- 


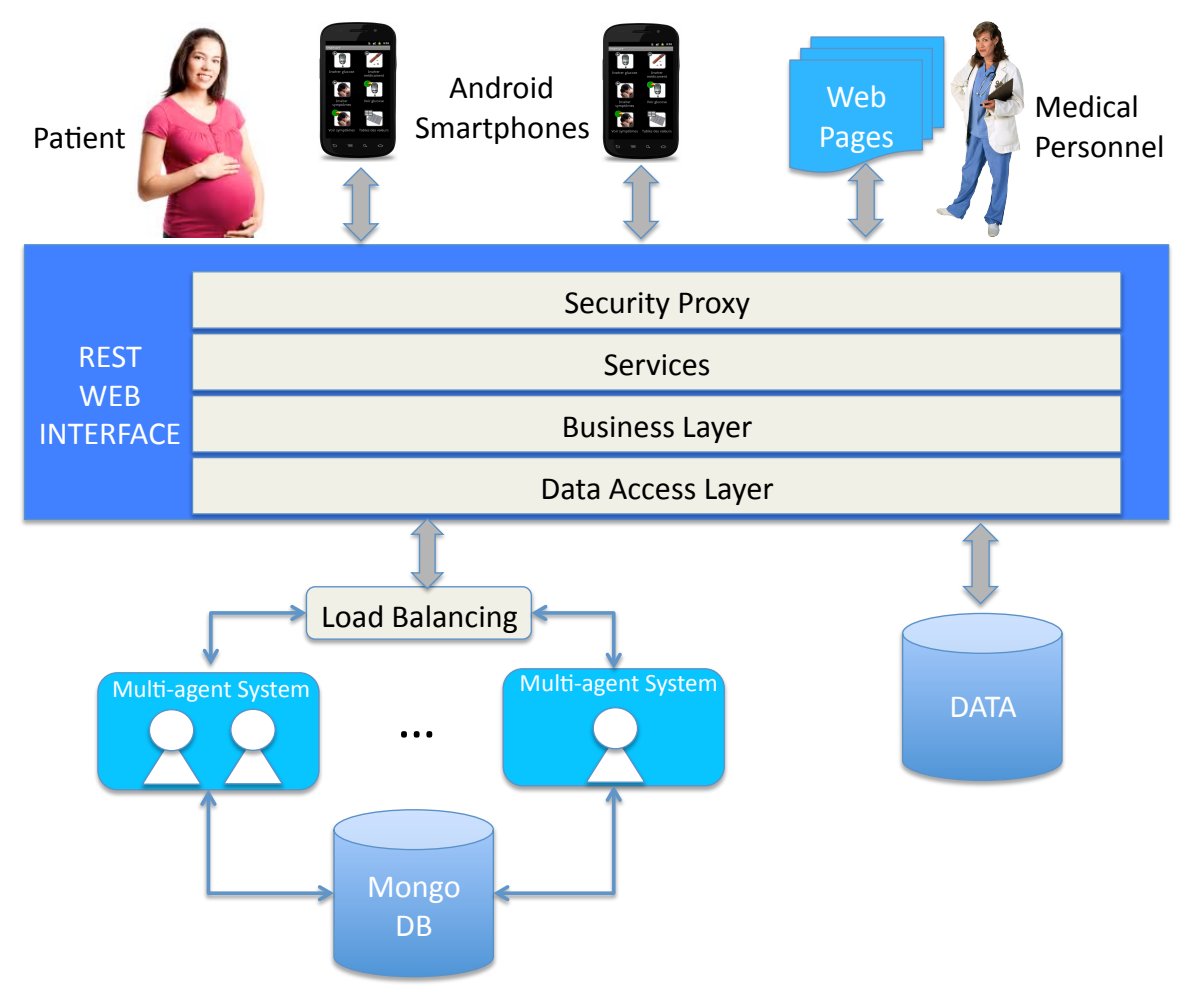

Fig. 1. Architecture of the Personal Health System to monitor GDM.

age within the smart phone again depends on the key stored in the QR code, consequently if the smart phone is stolen, without the QR code it is not possible to access the data of the use

Once authenticated, as shown in Fig. 3 a) the application provides the patient with a start page with a set of possible activies. Fig. 3 b), c), d), e) show respectively the interface that the patients would use to enter blood sugar measurements, the moment in the day when this was taken, the summary of blood measurements for the last week and a pie chart which shows the number of values which are too low, normal and too high. Values are too low if below $4 \mathrm{mmol} / \mathrm{l}$ and too high if above $5.3 \mathrm{mmol} / \mathrm{l}$ before a meal and above 7 $\mathrm{mmol} / \mathrm{l}$ if after a meal. This chart shows only the values stored on the phone for a week. This choice was made in agreement with medical doctors to mitigate situations where a stolen mobile phone would cause privacy issues. When entering blood sugar measurements, the mobile phone application will also look at the last inserted blood sugar values to preset the period. For ex- ample, if the last measurement was taken before dinner, an after dinner period will be preselected.

It is expected that patients enter their values in the smart phone after they took the measurement with the glucometer, thus by default, the current time is used for measurements, but patients can enter their blood sugar measurements at a later time.

Fig. $3 \mathrm{f}$ ) and g) show the interfaces to add medicaments taken and symptoms experienced.

For the medicine, patients can enter it with an associated amount. They can also indicate a reason for a deviation in an optional comment field.

Similarly, patients can introduce any symptoms they suffer from in the system. The symptoms shown are related to complications with GDM and have been selected by doctors. The day after patients will be asked if they still suffer from the symptom.

If a patient mistyped a measurement she can correct up to one week after entering the measurement. Once the user saves a value, the changed values are stored locally and synchronized with the server part of the system. Should there currently not be an active internet 

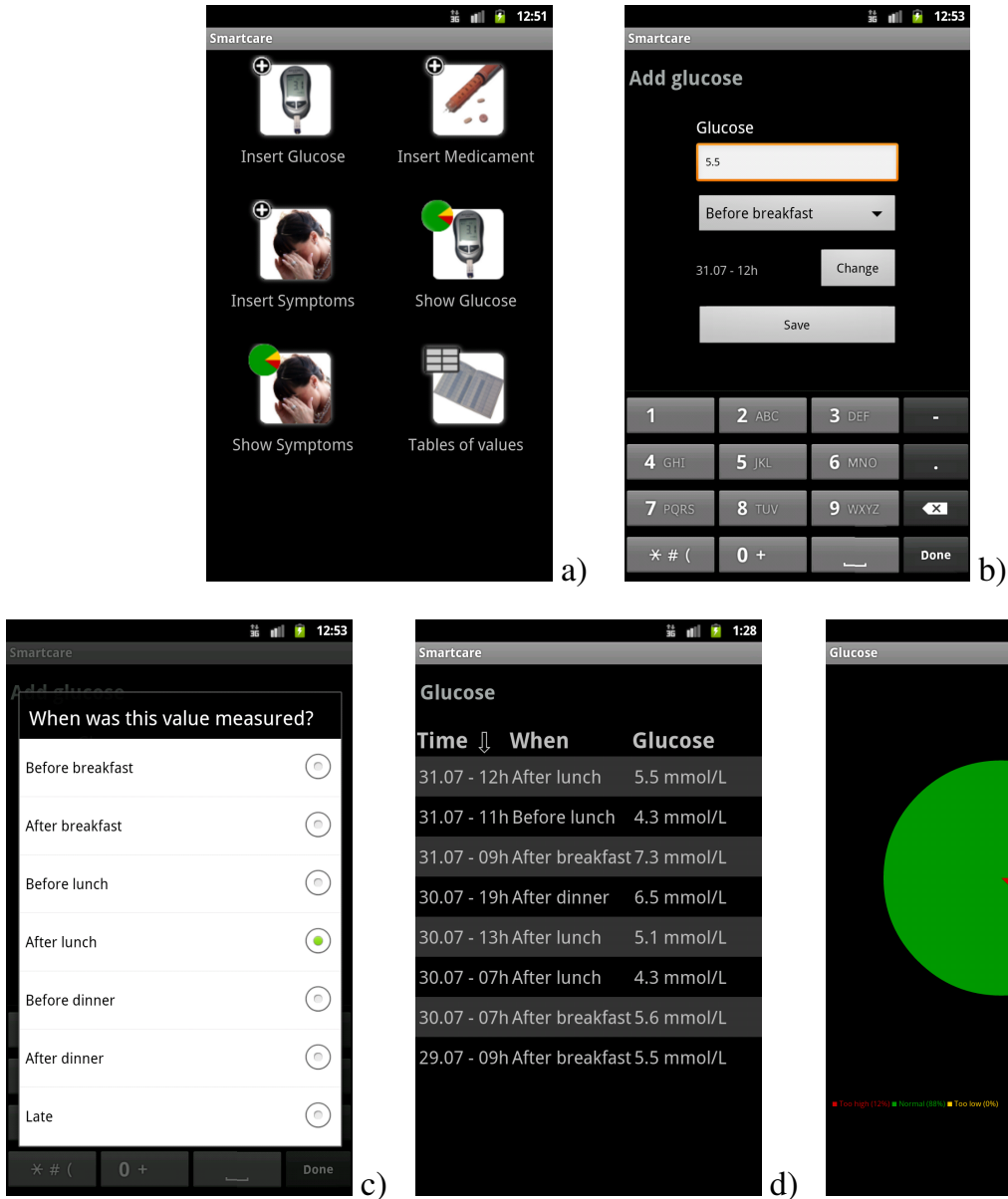

I: vill 1:28

Glucose

Time \. When Glucose

31.07- $12 \mathrm{~h}$ After lunch $5.5 \mathrm{mmol} / \mathrm{L}$

31.07 - $11 \mathrm{~h}$ Before lunch $4.3 \mathrm{mmol} / \mathrm{L}$

31.07 - 09h After breakfast $7.3 \mathrm{mmol} / \mathrm{L}$

30.07 - 19h After dinner $6.5 \mathrm{mmol} / \mathrm{L}$

30.07 - $13 \mathrm{~h}$ After lunch $\quad 5.1 \mathrm{mmol} / \mathrm{L}$

30.07 - 07h After lunch $\quad 4.3 \mathrm{mmol} / \mathrm{L}$

30.07 - 07h After breakfast $5.6 \mathrm{mmol} / \mathrm{L}$

29.07 - 09h After breakfast $5.5 \mathrm{mmol} / \mathrm{L}$

c)
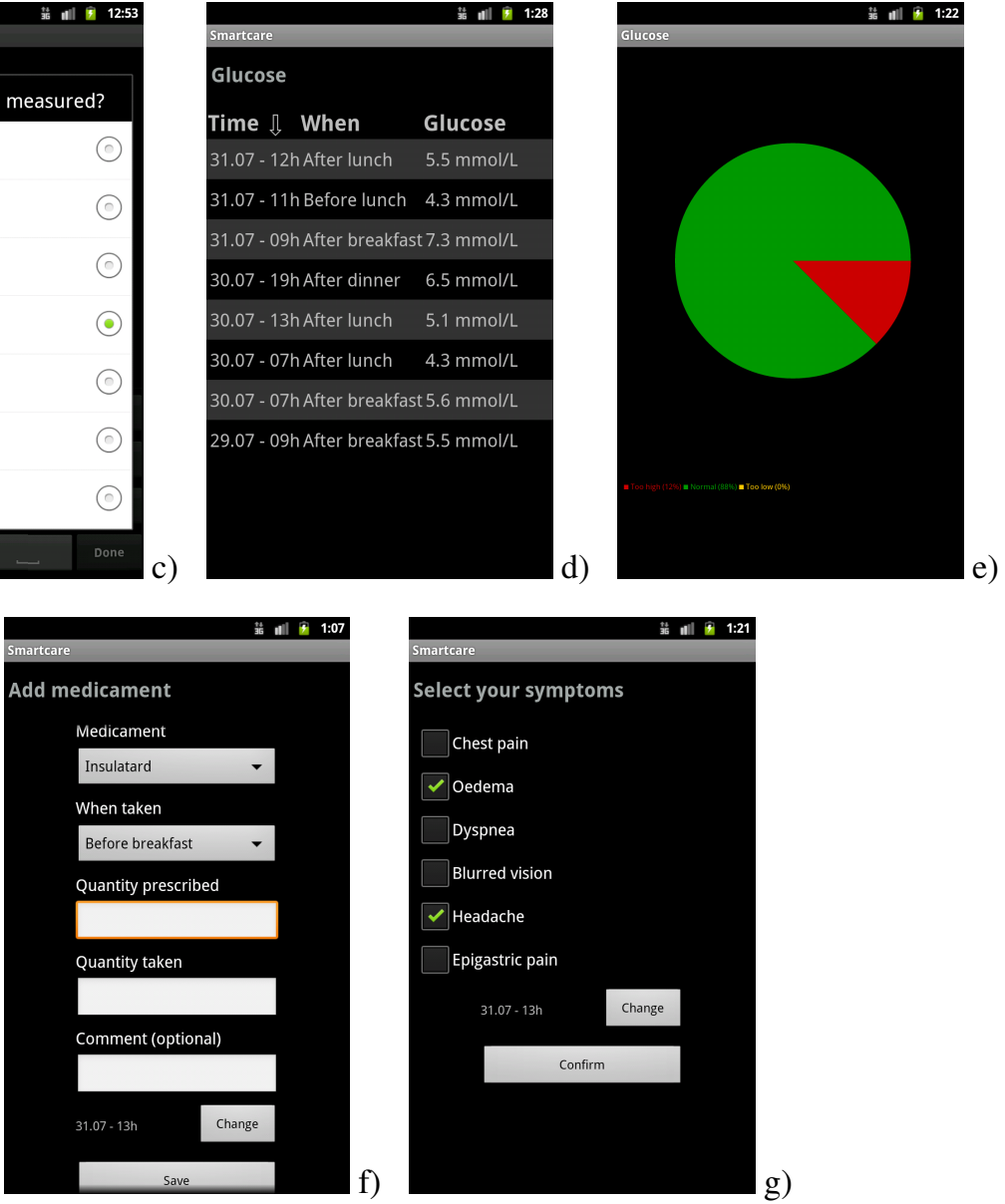

Fig. 3. Screens of the Android Interface. 
connection, the synchronization will be done on the next occasion.

\subsection{The Reasoning Agents}

Our system uses logic programming to deal with hyperglycaemia and hypoglycaemia detection during the treatment of the patient. A logic programming approach was selected as it is easy to interpret. The medical knowledge concerning glucose control in gestational diabetes was already available from our medical doctors in the University Hospital of Lausanne. The needs of our medical doctors was to enact this knowledge more efficiently at the patient's side, rather than learning a new model from the data. Another reason for this choice was the acceptability of the system from the perspective of the ethical commission: since the system can be interpreted with minimal effort by a human, a mistake or error is easier to spot and to explain than a statistical model, which may also be biased by a particular population.

Concerning the specific formalism used to represent the medical knowledge, we decided to use the Event Calculus (EC) [23]. In particular, we use a cached EC [14] version of the dialect of EC presented in [2], for performance reasons. EC is suitable to model expert systems representing the evolution in time of an entity, by means of the production of events. In this case, our EC reasoner models the behaviour applied by the medical doctors in the university hospital of Lausanne with respect to patients' glucose control. The events considered are the glycaemic events, the evolution in time of these values then trigger alerts to be reported to the medical doctors.

The reasoner is embedded in agents working as medical experts of GDM. In what follows we will use a Prolog like syntax, with lower case for predicates and upper case for variables. The symbol / is used to represent the arity (number of inputs) of a predicate.

Another possible choice to model the expert knowledge could have been the Arden syntax [20]. The main issue that we encountered with the Arden syntax was the lack of predicates to handle time evolution, and the fact that no indexing mechanism is considered, which implies that, even if we reimplemented EC with the Arden syntax, we would have problems when dealing with several hundred events, which we cannot solve by simply deleting the events from the knowledge base, as, being a mobile solution, it may happen that the events come at a later time or even unsorted in time.

\subsubsection{The Event Calculus}

EC is based on a many-sorted first-order predicate calculus represented as logic programs that are executable in Prolog. The underlying time model is linear. The EC manipulates fluents. A fluent represents a property which can have different values over time.

\begin{tabular}{|l|l|}
\hline Predicate & Meaning \\
\hline initially(F=V) & The value of fluent $\mathrm{F}$ is $\mathrm{V}$ at time 0. \\
\hline holds_at(F=V,T) & The value of fluent $\mathrm{F}$ is $\mathrm{V}$ at time $\mathrm{T}$. \\
\hline holds_for(F=V,[Tmin,Tmax] $)$ & $\begin{array}{l}\text { The value of fluent } \mathrm{F} \text { is } \mathrm{V} \text { between time } \\
\text { Tmin and time } \mathrm{Tmax} .\end{array}$ \\
\hline initiates_at(F=V, T $)$ & $\begin{array}{l}\text { At time } \mathrm{T} \text { the fluent } \mathrm{F} \text { is initiated to have } \\
\text { value } \mathrm{V} .\end{array}$ \\
\hline terminates_at(F=V, $\mathrm{T})$ & $\begin{array}{l}\text { At time } \mathrm{T} \text { the fluent } \mathrm{F} \text { is terminated from } \\
\text { having the value } \mathrm{V} .\end{array}$ \\
\hline broken(F=V, $[\mathrm{Tmin}, \mathrm{Tmax}])$ & $\begin{array}{l}\text { The value of fluent } \mathrm{F} \text { is either terminated } \\
\text { at Tmax, or initiated to a different value } \\
\text { than } \mathrm{V} \text { between Tmin and Tmax. }\end{array}$ \\
\hline happens_at(E,T) & $\begin{array}{l}\text { An event } \mathrm{E} \text { takes place at time } \mathrm{T} \text { updating } \\
\text { the state of the fluents }\end{array}$ \\
\hline
\end{tabular}

Table 1

EC with multi-valued fluents: predicates.

The term $F=V$, denotes that fluent $F$ has value $V$ that has been initiated by an action at some earlier time-point and not terminated by another action in the meantime. Tab. 1 summarizes the main EC predicates we use in this paper.

Predicates, function symbols and constants start with a lower-case letter while variables (starting with an upper-case letter) are universally quantified.

The specifications of the axioms of the EC are then represented below.

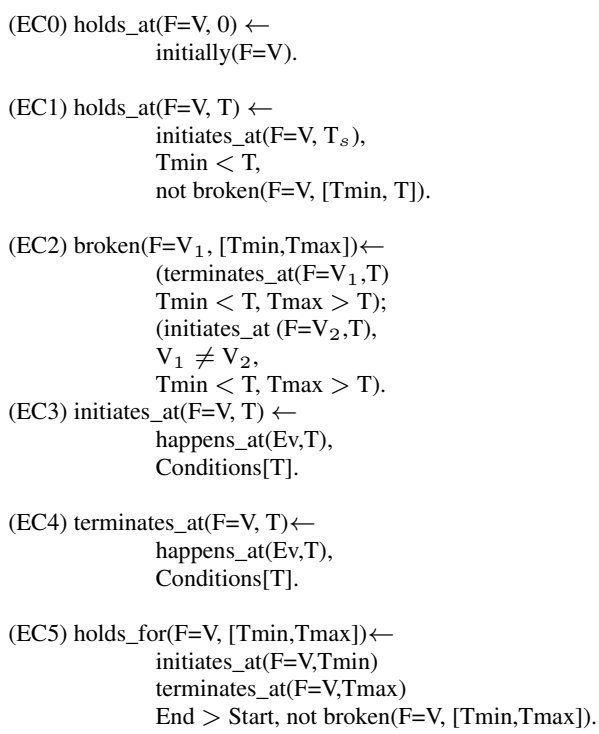

(EC4) terminates_at $(\mathrm{F}=\mathrm{V}, \mathrm{T}) \leftarrow$ happens_at(Ev,T), Conditions[T].

(EC5) holds_for(F=V, [Tmin,Tmax $]) \leftarrow$ initiates_at $(\mathrm{F}=\mathrm{V}, \mathrm{Tmin})$ terminates_at $(\mathrm{F}=\mathrm{V}, \mathrm{Tmax})$ End $>$ Start, not broken $(\mathrm{F}=\mathrm{V},[\mathrm{Tmin}, \mathrm{Tmax}])$.

(EC6) holds_for(F=V, [Tmin,infPlus] $) \leftarrow$ 


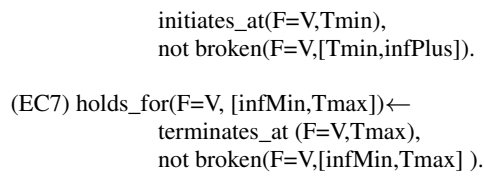

Clause EC0 states that a property $\mathrm{F}$ holds at time 0 if an intially/1 predicate is true at time 0 . Clause EC1 states that a property holds at a time $\mathrm{T}$ if it has been initiated at time Tmin and the holding of that property has not been broken between the starting time Tmin and the time of interest $\mathrm{T}$. To decide when a property is broken, we use the clause EC2. This states that a property $\mathrm{P}$ is broken between time Tmin and Tmax, if it is terminated at a time $\mathrm{T}$ between Tmin and Tmax or initiated to a different value between Tmin and Tmax. The other clauses specify when a property is initiated (EC3) or terminated (EC4), in terms of the conditions holding in the current context, typically expressed in terms of the holds_at $/ 2$, holds_for $/ 2$ predicates, meaning that such clauses will change according to the particular domain being modeled with the EC. EC5-EC7 express the EC clauses that deals with validity intervals of fluents. In particular, EC5 specifies that a fluent F keeps a value $V$ for an interval going from Tmin to Tmax if nothing happens in the middle that breaks such an interval. EC6-EC7 behave like EC5, but deal with open intervals.

\subsubsection{Medical Symbolic Reasoning in the EC}

Given the patient glucose measurements during pregnancy and the targets specified above, the medical doctors involved in the study apply the following rules in their practice (see [33] for an additional reference), before changing the patient's treatment:

- if two glucose values are less than $4 \mathrm{mmol} / \mathrm{l} \mathrm{mea-}$ sured at the same time of day on two consecutive days in insulin dependent patients then the doctor should react to contain hypoglycaemia events.

- if two glucose values are less than $3 \mathrm{mmol} / \mathrm{l}$ within one hour then this is a serious hypoglycaemia and the doctor should be informed immediately.

- if fasting glucose is bigger than $5.8 \mathrm{mmol} / \mathrm{l}$ on any two days of the four preceding days then the doctor should intervene to limit hyperglycaemia events in the morning.

- if fasting glucose is bigger than $5.3 \mathrm{mmol} / \mathrm{l}$ on any three days of the five preceding days the doctor should intervene to limit hyperglycaemia events in the morning.
- if postprandial glucose is bigger than $8 \mathrm{mmol} / \mathrm{l}$ measured at the same time of day on any two days of the preceding four days or it is bigger than 7 $\mathrm{mmol} / \mathrm{l}$ measured at the same time of day on any four days of the seven preceding ones then the doctor should intervene to limit postprandial hyperglycaemia events.

The treatment consists in doses of slow insulin, an insulin acting slowly during the day or during the night, or fast insulin, an insulin acting immediately and whose effect lasts only for a couple of hours. For example, if a patient experiences a glucose above 7 $\mathrm{mmol} / \mathrm{l}$ three times in a row during postprandial dinner, then the medical doctors should introduce (or increase) fast insulin before dinner.

We can now define monitoring rules for a GDM affected woman using the EC dialect previously presented. In defining these rules, our goal will be to report quickly situations where the doctor should react as we defined them above.

To deal with the monitoring of our patients, we utilize the GOLEM agent platform [10,24,11,9]. Such a platform deals with the representation of events in EC, which then are notified to our logic based agents, whose architecture is depicted in Fig. 4. The agents deployed in GOLEM are active entities and their reasoning cycle is expressed by two control threads, one to receive the events notified to the agent in the agent environment (called perception_cycle/1) and one to produce actions in the agent environment (called action_cycle/1).

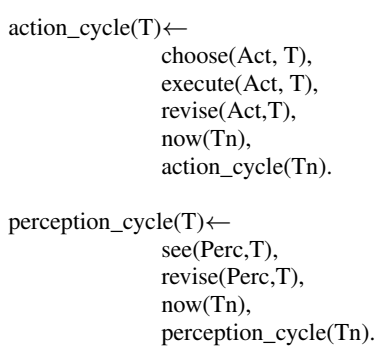

The two cycles described above, utilize then the Treatment Adjustment Deductive Rules module to produce alerts for the medical doctors in charge of the patients. The choose/2 predicate is specified below:

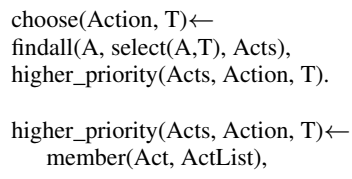




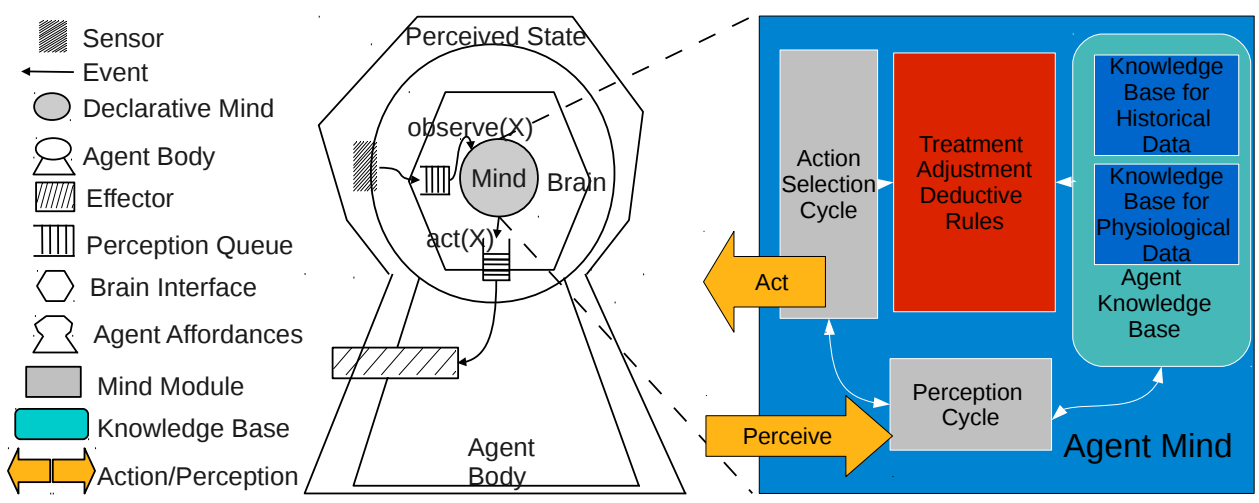

Fig. 4. Caretaker Agent Mind Architecture.

priority(Act, $\mathrm{P}, \mathrm{T})$,

not (member(ActX, ActList),

not ActX = Act,

priority(ActX, PX,T), PX > P).

select(submit_alert(AlertType,Period), T) $\leftarrow$

holds_at(alert(AlertType,Period)=true,T).

priority(submit_alert(serious_hypoglycaemia,_),P,T) $\leftarrow$ holds_at(serious_hypoglycaemia=priority $(\mathrm{P}), \mathrm{T})$.

priority(submit_alert(hypoglycaemia,_),P,T) holds_at(hypoglycaemia=priority $(\mathrm{P}), \mathrm{T})$.

Such a predicate finds all the possible actions that can be executed by the agent and then performs the one with the highest priority. The specification of the priority/ 3 predicate is shown above for two different alerts associated with hypoglycaemia.

In particular, within our logic based agents, we defined domain initiates_at/2 rules to deal with the evolution of the glucose of the patients during the week.

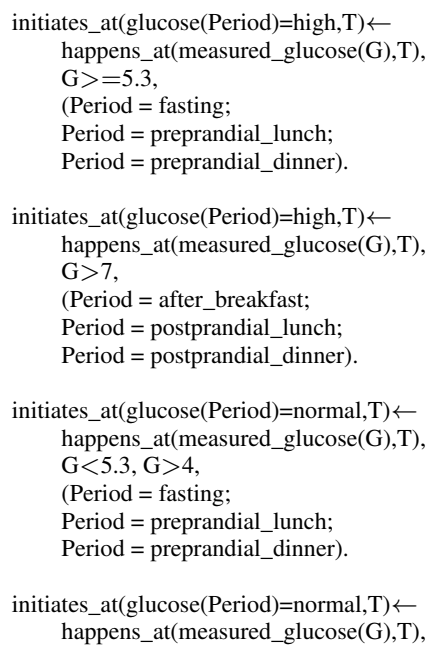

$\mathrm{G}<7, \mathrm{G}>4$,

$($ Period $=$ after_breakfast;

Period $=$ postprandial_lunch;

Period $=$ postprandial_dinner) .

initiates_at $($ glucose $($ Period $)=$ low,$T) \leftarrow$ happens_at(measured_glucose $(\mathrm{G}), \mathrm{T})$,

$\mathrm{G}<4$.

The initiates_at/2 rules above specify when the glucose of the patient is considered high. The predicate period_of_day/2 returns the period of the day when the glucose result is produced.

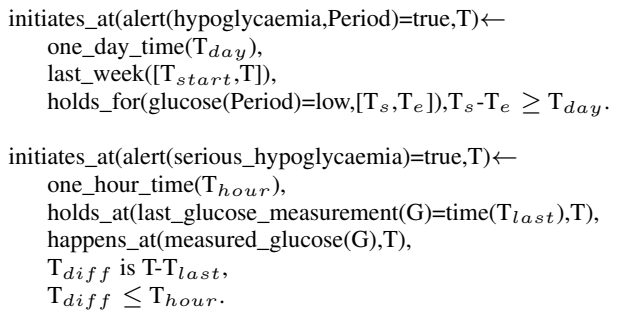

The initiates_at/ 2 above specifies that hypoglycaemia alerts are triggered in two cases. In the first case, the woman is experiencing recurrent episodes of hypoglycaemia in the same period of the day and then the alert is triggered. The second alert is triggered when the patient has twice a hypoglycaemia within one hour of time, which is considered as a serious hypoglycaemia that needs immediate consideration from the doctors.

Similarly, we define rules to handle hyperglycaemia. Considering the medical guidelines followed by our medical partners, we modeled the following EC rules for postprandial hyperglycaemia events.

initiates_at(alert(postprandial_hyperglycaemia)=active, $\mathrm{T}) \leftarrow$ happens_at $\left(g l u c o s e\left(\mathrm{~V}_{1}, \mathrm{P}\right), \mathrm{T}\right)$, last_four_days(Time4days,T), 


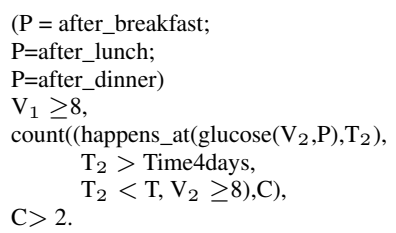

initiates_at(alert (postprandial_hyperglycaemia $)=$ active, $T) \leftarrow$ happens_at (glucose $\left.\left(\mathrm{V}_{1}, \mathrm{P}\right), \mathrm{T}\right)$,

last_week(Time7days, T)

$(\mathrm{P}=$ after breakfast;

$\mathrm{P}=$ after_lunch;

$\mathrm{P}=$ after_dinner)

$\mathrm{V}_{1} \geq 7$,

count((happens_at(glucose $\left.\left(\mathrm{V}_{2}, \mathrm{P}\right), \mathrm{T}_{2}\right)$,

$\mathrm{T}_{2}>$ Time7days,

$\mathrm{C}>3$.

$\left.\left.\mathrm{T}_{2}<\mathrm{T}, \mathrm{V}_{2} \geq 7\right), \mathrm{C}\right)$
$\mathrm{T}_{2}$

Where with count/2 we specify the amount of times the condition taken in consideration holds. The first rule states that if a patient had hyperglycaemia during postprandial periods for twice in the last four days with a value of glucose above $8 \mathrm{mmol} / \mathrm{l}$, or three times in the last week with a value above $7 \mathrm{mmol} / \mathrm{l}$, then an alert of hyperglycaemia shall be fired so that the doctor can check the diet and treatment of the GDM patient.

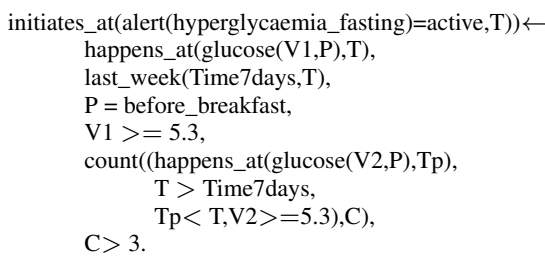

initiates_at(alert(hyperglycaemia_fasting $)=$ active, $T)) \leftarrow$ happens_at (glucose $(\mathrm{V} 1, \mathrm{P}), \mathrm{T})$,

last_four_days(Time4days,Tcall),

$\mathrm{P}=$ before_breakfast

Vall $>=5.8$,

count((happens_at(glucose(V2,P),Tp),

$\mathrm{T}>$ Time4days,

$\mathrm{C}>2$.

$\mathrm{Tp}<\mathrm{T}, \mathrm{V} 2>=5.8), \mathrm{C})$

Furthermore, in gestational diabetes it is of particular interest to control the hyperglycaemia before breakfast in order to decide whether or not the patient should take a long lasting insulin at night before going to sleep. The two rules above trigger an alert in the case the patient is repeatedly having glucose values above $5.3 \mathrm{mmol} / \mathrm{l}$ or $5.8 \mathrm{mmol} / \mathrm{l}$ at fasting.

\subsection{Doctor's Web Interface}

Medical caretakers interact with the system using a web based application to consult the events produced by the mobile phones or the alerts produced by the agents.

After login the user has access to the start page as shown on the top of Fig. 5. From this page the caregiver can have access to the patient's page by clicking on a patient's name, as shown on the bottom of Fig. 5. The patient's page shows alerts for patients associated with the caretaker. These alerts are sorted by date, showing the most recent alerts first.
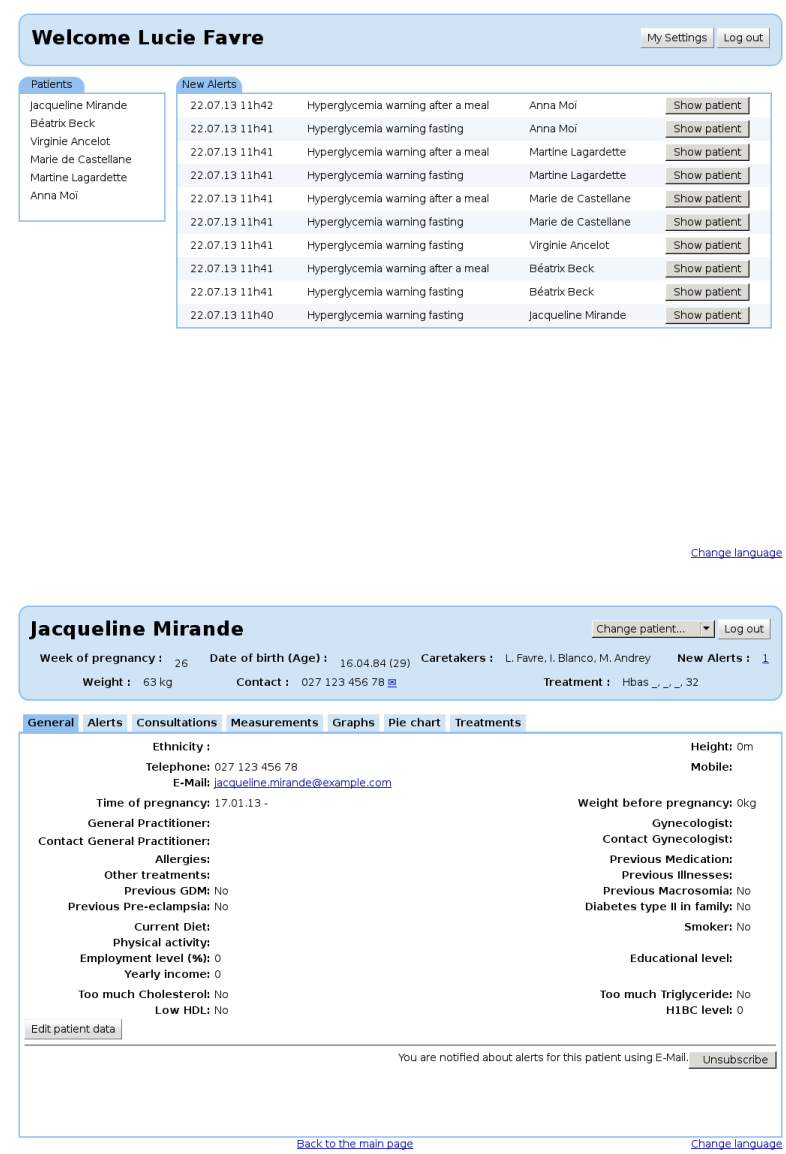

Fig. 5. Web Application: Start Page and Patient's Page.

The patient's page is used to view, modify and visualize all patient information in terms of: the patient's name, week of pregnancy, date of birth and age, which caretakers are associated with the patient, alerts, the current weight, contact information (telephone and email) and the current treatment. The content pages accessible from the patient's page are: general patient information, alerts for the patient, consultations with the patient, physiological values of the patient, a graphical 
representation of the patient's physiological values in the form of graphs, a higher level graphical representation of the patient's blood sugar values in the form of pie charts and the treatments of the patient.

The general patient information content page is used to show patient contact information, factors which influence gestational diabetes and demographic information. Factors which influence the course of a patient's GDM such as the current diet or any allergies as well as risk factors for conditions like preeclampsia are the second part of the general patient information content page. Lastly, demographic information are collected due to suspected relations with GDM.

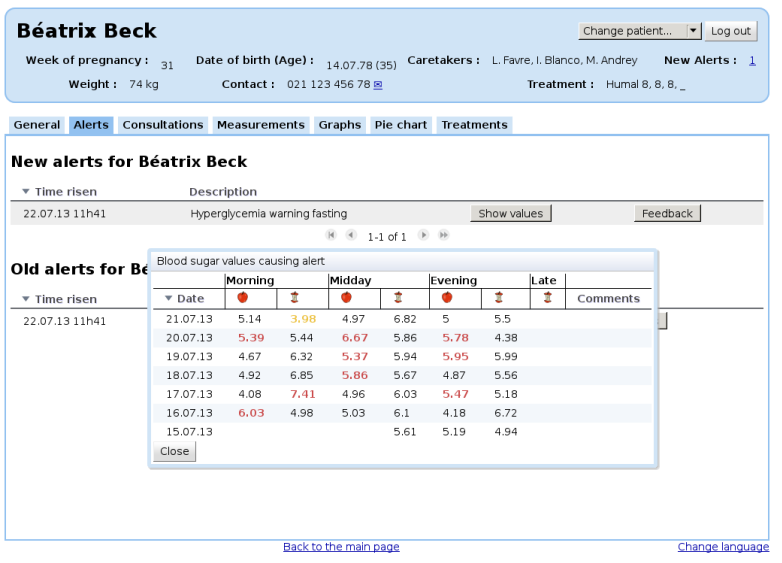

Fig. 6. Web Application: Alerts Interfaces

The patient's alerts content page is shown in Fig. 6 . In order to evaluate an alert, the caretaker can see the physiological values which lead towards raising the alert.

The consultations content page visible in Fig. 7 allows tracking the contacts between caregivers and patients. Consultations can be done either in person or using a telephone. In addition to the moment, type, duration, reason for the consultation, an optional comment can be given.

Physiological values measured by the patient as well as any medicine taken are shown on the measurements content page (Fig. 7) in a tabular format. The values for blood sugar and medicine are presented for every day in relation to each meal and before going to bed, with associated comments if present.

Clicking on a day in the blood sugar and medicine table will open the detailed day view which can be seen on the bottom of Fig 7. This view shows each measurement introduced by the patient in the phone in relation
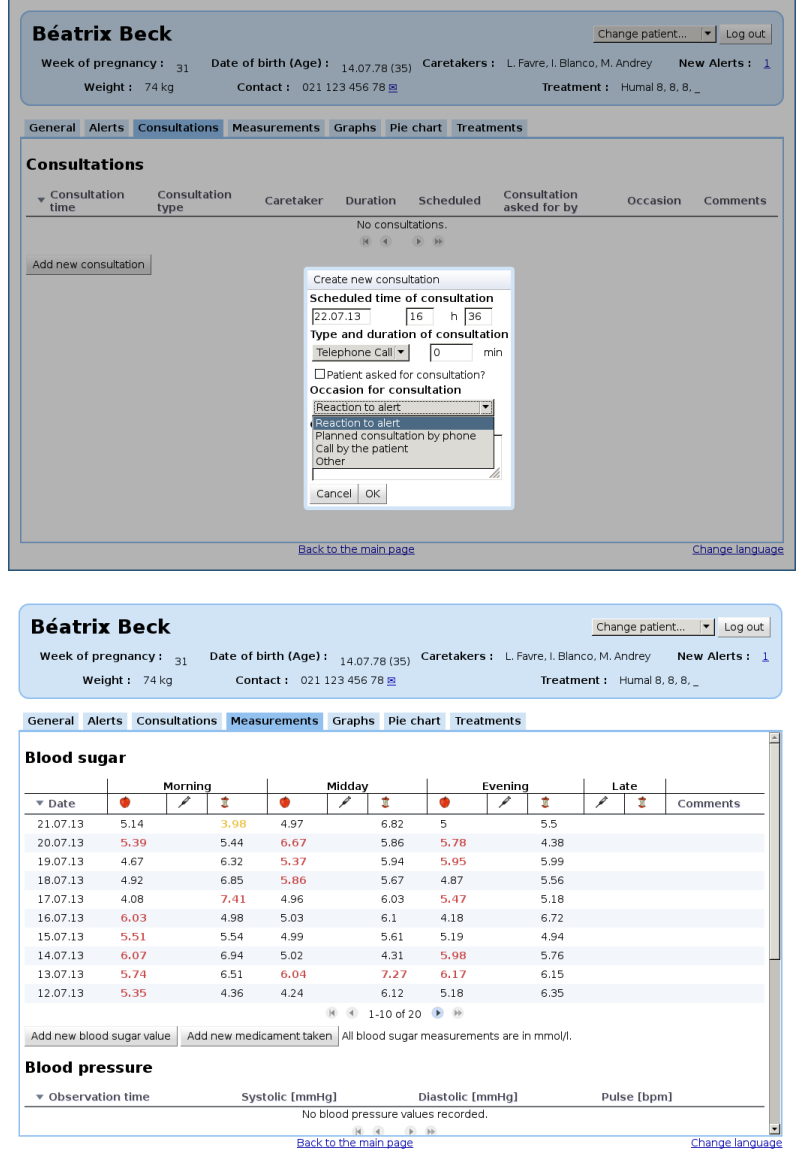

Fig. 7. Consultations Content Page.

to a meal and medicine taken. Caretakers can comment on values.

Caretakers can correct or add patient's values by clicking on a measurement in the detailed day view. A caretaker can similarly modify or create entries for medicines taken by a patient.

The graph content page, as shown in Fig. 8, allows caretakers to visualize a patient's physiological values.

The graph itself shows a user selectable period of one week, one month or three months for a patient starting on a user selectable starting date. The graph has background lines to show the borders between days and depending on the shown period also every 6 hours. Weekends are highlighted with a subtle grey background. For blood sugar the normal range of 4 to 7 $\mathrm{mmol} / \mathrm{l}$ is highlighted with a subtle yellow background color to permit easily spotting values above or below the normal range. The displayed physiological values are drawn in different colors for breakfast, lunch, din- 


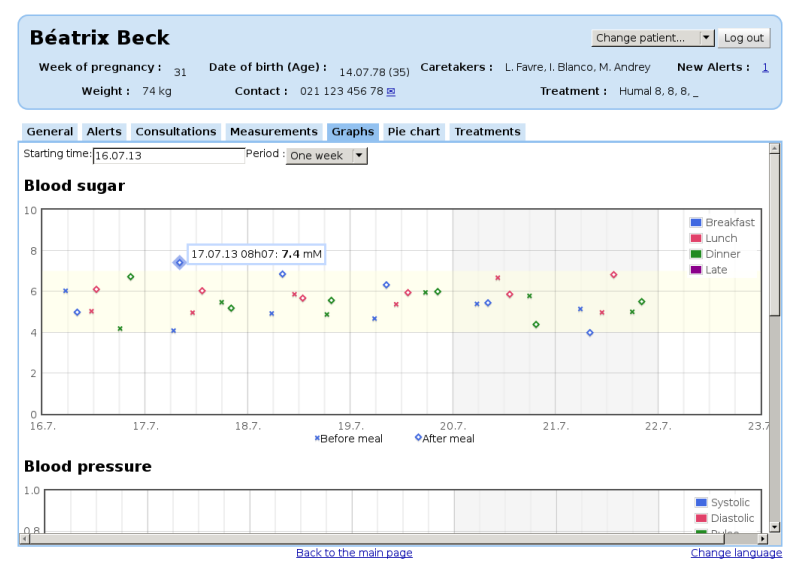

Fig. 8. Graph Representation of Values.

ner and measurements before going to bed. Measurements before or after a meal are furthermore shown using differing symbols: A cross symbol for values before a meal and a diamond symbol for values after a meal.

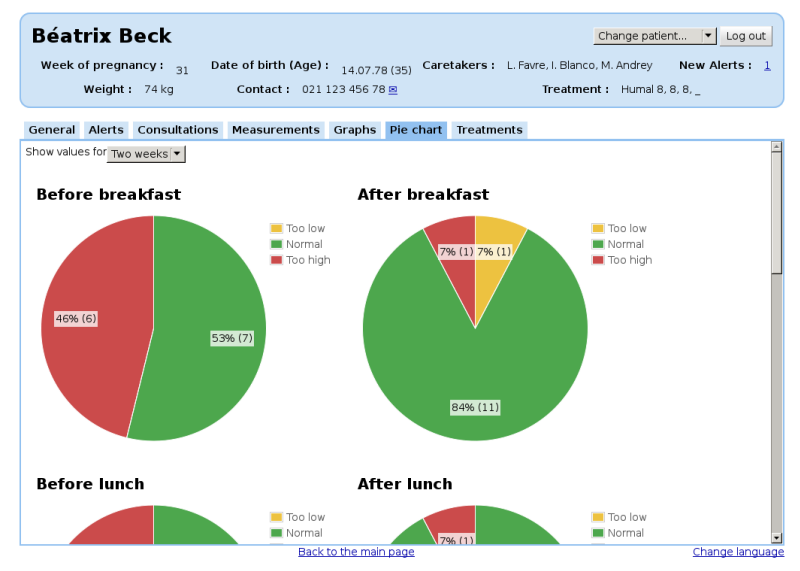

Fig. 9. Pie chart representation.

While the graph representation has been designed in collaboration with caretakers to present a useful visualization of values for caretakers, it is more difficult to understand for patient and therefore less useful as a communication aid for a caretaker when talking to a patient. The pie charts content page visible in Figure 9 has been designed to help caretakers explain a patient their values in an easy way.

Patients' blood sugar values are classified as too low, normal and too high and then displayed in a pie chart: red slices represent values which are too high, yellow slices represent values which are too low and normal values are colored in green.

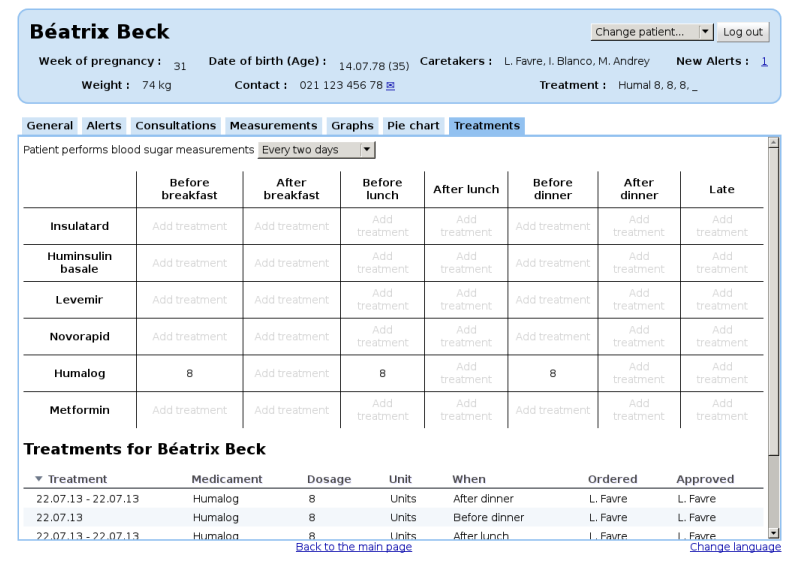

Fig. 10. Treatments Table.

The last content page is the treatments content page as seen in Fig. 10. This page allows caretakers to input the amount of medicine a patient should take at a certain time.

All treatments including their duration are shown in a tabular form below the table used for user input. Since only doctors are allowed to prescribe a medicine to a patient, nurses have to confirm that they spoke with a doctor before a value is saved. This allows to track who is ultimately responsible for a prescription.

Generally speaking, a doctor is only allowed to access patient data if a treating relationship exists between them. User's permissions are determined by their group membership and treatment relationships.

To authenticate the caregivers when accessing our system from the Web, we utilize user name, password and a certificate as the two authentication means. The certificate is stored on a smart card.

The interface specified for server side of the PHS was built following the requirements specified by our medical doctors. The data visualization Web interfaces have been kept simple on purpose. The medical doctors involved in the study wanted to be able to explain the results obtained to their patients during the consultations. As a consequence a simple representation of the values in terms of pie charts and point clouds was preferred. Another reason not to present elaborated graphs that would show trends, was not to bias the judgement of the caregivers during the treatment, as the collection of the glucose values was not done us- 
ing a Continuous Glucose Monitor and the pattern of glucose could vary significantly in between the sampling done using the standard glucometer provided to the patients.

In addition, the interface to present the glucose values to the patients was explicitly modelled following the same fashion of the notepad that the patients use in the standard treatment, in order to be able to better explain problems with the glucose control. A secondary reason for this modelling was also to simplify the acceptability of the system to the medical doctors that would not have to modify their way of interacting with the patients thanks to the similarity of the system with the standard material.

\section{Feasibility Study Evaluation}

The evaluation of our system has been performed by means of a feasibility study, taking the form of a single-center, parallel group, open randomized controlled trial conducted at Lausanne University Hospital. Screening for study inclusion was performed from February 2013 to June 2013 in all patients suffering from GDM and treated at the University Hospital of Lausanne. Inclusion criteria comprised the ability to speak French and diagnosis of GDM within $>24$ th and $<32$ nd gestational week. All patients had to provide written informed consent prior to study inclusion. Patients were assigned to 2 different groups: standard protocol group (SP) and telemedicine care group (TM). Follow-up was performed from the 2nd clinic visit to childbirth in every patient. SP patients were subjected to usual follow-up, which comprised regularly scheduled clinic visits and recording of glucose values in a notebook. TM patients were managed by our PHS system and regularly scheduled clinic visits. In this trial, the PHS system was integrated on a Samsung GT-S5570 Galaxy Smartphone. The targeted feasibility outcome was whole trial feasibility, functioning of the PHS and its appropriateness for patient use. The 2 groups were compared using univariate quantitative analysis. Additionally, qualitative analysis was performed for patients treated by telemedicine. The scope of this feasibility trial was to assess the feasibility and acceptability of our PHS in patients suffering from GDM. The trial was performed to determine optimal frame conditions for a subsequent main trial given the feasibility end point could be achieved. Furthermore, the derivation of parameters for the aforementioned main trial comparing our PHS to standard care such as recruitment- and follow-up rates, glucose control medical complications in mother and newborn, and health care costs, was another scope of this trial. Hypothesis testing and subsequent inference derived from the statistical analyses of the secondary end points is not an objective of the present trial.

Table 2 shows the demographics of the patients in the two arms of the trial.

\subsection{Glucose Control Results}

There were a mean of $185 \pm 96$ recorded glucose values per patient (Table 2a). Patients in the TM group recorded significantly more glucose values $(235 \pm 86)$ than patients in the SP group (135 \pm 80$)$.

Table 3 displays median [IQR] of the 6 daily measurements for plasma glucose values. For fasting glucose in the morning, TM patients showed a median [IQR] of 4.6mmol/1 [4.3-4.9] and SP patients showed a median [IQR] of 4.8mmol/1 [4.5-5.2], respectively $(\mathrm{p}<0.001)$. For postprandial glucose at noon SP patients had $\mathrm{SP}=6.3 \mathrm{mmol} / 1$ [5.6-7.3] vs. telemedicine group $\mathrm{TM}=5.9 \mathrm{mmol} / 1$ [5.2-6.6] $(p<0.001)$. Most, i.e. 4 out of 6 , of the daily capillary glucose measurement were significantly better controlled with telemedicine care. The variance of capillary glucose measurements was equally lower in the TM than in the SP group. Hypoglycaemic episodes occurred with a median [IQR] of 0.5 [0-4.75] and $3.5[0-8.75]$ in the $\mathrm{SP}$ and the TM group respectively. Patients in the SP group experienced $30 \pm 30$ hyperglycaemic episodes and patients in the TM group $31 \pm 23$ ( $\mathrm{p}=0.88$ ). Insulin was prescribed to 7 (29\%) patients totally. Two patients $(8 \%)$ in each group received basal insulin. Bolus insulin was prescribed to $3(13 \%)$ patients overall, 2 of whom belonged to the TM and 1 to the SP group. Furthermore, $2(8 \%)$ patients in the SP group were prescribed Metformin.

\subsection{Alerts Results}

Alerts pertain only to patients treated by telemedicine care. For hyperglycaemia, alerts were issued $19 \pm 12$ times per patient. Hyperglycaemic episodes and issued alerts showed a strong, positive and significant correlation between $(\mathrm{r}=0.86, \mathrm{R} 2=0.74, \mathrm{p}<0.001)$ (Fig. 11). Hypoglycaemia alerts occurred only 7 times and were issued in 3 patients. 
Table 2

Baseline Patient Characteristics.

\begin{tabular}{|c|c|c|c|c|}
\hline & Overall $(\mathrm{N}=24)$ & Standard Protocol $(\mathrm{N}=12)$ & Telemedicine $(\mathrm{N}=12)$ & p-value \\
\hline Age, years $\pm S D$ & $32 \pm 5$ & $32 \pm 4$ & $33 \pm 5$ & 0.45 \\
\hline Weight, kg士SD & $80.8 \pm 20$ & $82.2 \pm 21.6$ & $79.4 \pm 19.1$ & 0.74 \\
\hline BMI, kg2 \pm SD & $30.0 \pm 6.9$ & $30.7 \pm 7.9$ & $29.2 \pm 5.9$ & 0.61 \\
\hline Arterial blood pressure, $\mathrm{mmHg} \pm \mathrm{SD}$ & $119 / 75 \pm 10 / 9$ & $115 / 71 \pm 8 / 8$ & $123 / 78 \pm 11 / 9$ & $0.06 / 0.05$ \\
\hline $\mathrm{HbA} 1 \mathrm{c}, \% \pm \mathrm{SD}$ & $5.3 \pm 0.3$ & $5.3 \pm 0.3$ & $5.2 \pm 0.4$ & 0.47 \\
\hline GW at study inclusion, mean \pm SD & $29.1 \pm 1.9$ & $29.2 \pm 1.5$ & $28.9 \pm 2.2$ & 0.67 \\
\hline \multicolumn{5}{|l|}{ Patient history } \\
\hline Gestity, median [IQR] & $2.5[1-4]$ & $3[2-4]$ & $1.5[1-3.5]$ & 0.16 \\
\hline Parity, median [IQR] & $0[0-1.5]$ & $1[0-3]$ & $0[0-0.5]$ & 0.06 \\
\hline Dyslipidemia, n(\%) & $1(4)$ & $1(8)$ & $0(0)$ & 1 \\
\hline Cardiovascular disease, $\mathrm{n}(\%)$ & $1(4)$ & $0(0)$ & $1(8)$ & 1 \\
\hline Hypertension, $\mathrm{n}(\%)$ & $0(0)$ & $0(0)$ & $0(0)$ & 1 \\
\hline Previous GDM & $1(4)$ & $0(0)$ & $1(8)$ & 1 \\
\hline \multicolumn{5}{|l|}{ Family history } \\
\hline Dyslipidemia, n(\%) & $4(17)$ & $2(17)$ & $2(17)$ & 1 \\
\hline Cardiovascular disease, $\mathrm{n}(\%)$ & $6(25)$ & $5(42)$ & $1(8)$ & 0.16 \\
\hline Hypertension, $\mathrm{n}(\%)$ & $9(38)$ & $4(33)$ & $5(42)$ & 1 \\
\hline Obesity, $\mathrm{n}(\%)$ & $4(17)$ & $2(17)$ & $2(17)$ & 1 \\
\hline \multicolumn{5}{|l|}{ Diabetes, $\mathrm{n}(\%)$} \\
\hline Citizenship & & & & 0.24 \\
\hline Europe & $18(75)$ & $7(58)$ & $11(92)$ & \\
\hline Africa & $3(13)$ & $2(17)$ & $1(8)$ & \\
\hline South America & $2(8)$ & $2(17)$ & $0(0)$ & \\
\hline Australia & $1(4)$ & $1(8)$ & $0(0)$ & \\
\hline Place of birth & & & & 0.14 \\
\hline Europe & $17(71)$ & $6(50)$ & $11(92)$ & \\
\hline Africa & $4(17)$ & $3(25)$ & $1(8)$ & \\
\hline South America & $2(8)$ & $2(17)$ & $0(0)$ & \\
\hline Australia & $1(4)$ & $1(8)$ & $0(0)$ & \\
\hline \multicolumn{5}{|l|}{ Socioeconomic } \\
\hline Martial status & & & & 0.44 \\
\hline Single, $\mathrm{n}(\%)$ & $9(38)$ & $3(25)$ & $6(50)$ & \\
\hline Married, $\mathrm{n}(\%)$ & $12(50)$ & $7(58)$ & $5(42)$ & \\
\hline Divorced, $\mathrm{n}(\%)$ & $3(12)$ & $2(17)$ & $1(8)$ & \\
\hline Economical sector of occupation & & & & 0.24 \\
\hline Primary, $\mathrm{n}(\%)$ & $4(17)$ & $3(25)$ & $1(8)$ & \\
\hline Secondary, n(\%) & $8(33)$ & $5(42)$ & $3(25)$ & \\
\hline Tertiary, $\mathrm{n}(\%)$ & $12(50)$ & $4(33)$ & $8(67)$ & \\
\hline Physiotherapy, n(\%) & $9(38)$ & $3(25)$ & $6(50)$ & 0.4 \\
\hline
\end{tabular}


Table 3

Glucose Control Results. ${ }^{*}$ measurements in mmol/1 and median [IQR: 25\%-75\%]. ${ }^{o}$ p-values are from independent samples Mann-Whitney U test. ${ }^{*}$ p-values are from variance ratio test.

\begin{tabular}{|c|c|c|c|c|}
\hline Plasma Glucose measurements* & Standard Protocol $(\mathrm{N}=12)$ & Telemedicine $(\mathrm{N}=12)$ & p-value ${ }^{O}$ & p-value for variance* \\
\hline Nb of measurements & 1616 & 2749 & & \\
\hline Overall glucose control & $5.7[4.9-6.7]$ & $5.4[4.7-6.4]$ & $<0.001$ & $<0.001$ \\
\hline Nb of measurements & 485 & 723 & & \\
\hline Fasting & $4.8[4.5-5.2]$ & $4.6[4.3-4.9]$ & $<0.001$ & $<0.001$ \\
\hline Postprandial & $6.1[5.3-6.8]$ & $5.7[4.9-6.5]$ & $<0.001$ & $<0.01$ \\
\hline \multicolumn{5}{|l|}{ Noon } \\
\hline Nb of measurements & 83 & 46 & & \\
\hline Fasting & $5.4[4.7-6.4]$ & $5[4.2-6.1]$ & 0.08 & $<0.05$ \\
\hline Nb of measurements & 368 & 651 & & \\
\hline $\mathrm{Nb}$ of measurements & 26 & 100 & & \\
\hline Fasting & 4.7 & {$[4.3-5.5]$} & $<0.001$ & $<0.001$ \\
\hline Nb of measurements & 406 & 692 & & \\
\hline Postprandial & 6.3 & {$[5.6-7]$} & 0.59 & $<0.001$ \\
\hline
\end{tabular}

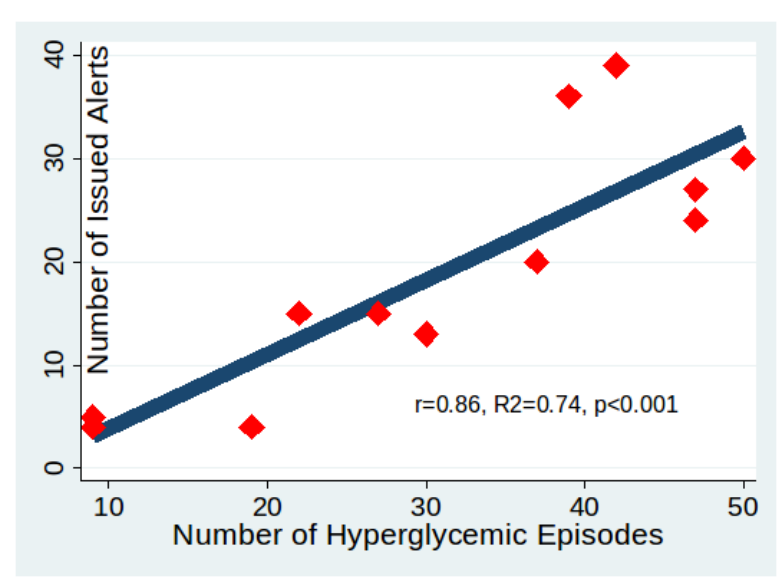

Fig. 11. Scatterplot with regression line displaying the significant relationship between hypeglycaemic episodes and issued alerts by the PHS.

\subsection{Qualitative Analysis}

To perform a qualitative data analysis, we provided questionnaires to patients and doctors to assess their experience with the system. For patients the questionnaires focused on assessing the acceptance and perception of the technology from the perspective of the patients. Similarly, for the medical doctors the questionnaire assessed their perception of the technology from the perspective or reduction or increase of workload. Finally, we also run a focus group to assess the opinions, beliefs and attitudes of the healthcare providers concerning the telemedicine technology.

In the telemedicine protocol, eleven out of 12 questionnaires were returned. The patient who did not provide the questionnaire suffered from a recurrent depressive disorder she had not declared prior to study inclusion and would not respond to our contact attempts. All patients were at least satisfied with the care provided. $73 \%$ of patients were very satisfied. The data entry and the usage of the smartphone was rated by all patients $(100 \%, \mathrm{n}=11)$ as easy ( $64 \%$ as very easy). The latter equally indicated to have received sufficient information regarding the use of the smartphone $(100 \%$, $\mathrm{n}=11$ ). The telemedicine technology conferred an additional impression of safety to $91 \%$ of patients.

Telemedicine technology was considered by the four caregivers involved as appropriate for the management of patients suffering from GDM, but they did not feel it implied less consultations with the patient. On the positive side, the medical staff involved is of the opinion that two factors were promising: one was the improvement of the quality of the treatment given by the possibility of consulting the patient's values day by day, and secondly the time saved by the fact that alerts would allow the doctors to focus on hyperglycaemic 
or hypoglycaemic events, rather than having to go through two weeks of data written on a notebook. Another positive aspect that was pointed out by the caregivers concerns the reaction time: thanks to the reporting of the GDM monitoring system, the telemedicine protocol reaction time to change the medicament of a patient that has a poor glycaemic control was one to three days against the one to three weeks (depending on the rate of consultation) required for the standard protocol, which is also confirmed by the better glycaemic control achieved by the telemedicine group as compared to the standard group.

\section{Related Work}

The problem of monitoring patients affected by gestational diabetes using telemedicine technology has been previously studied $[15,26]$, showing positive results from the perspective of the satisfaction of the patients and the outcome of the pregnancy. In general terms, thanks to the advancements of telecommunication technology, we can find a proliferation of telemedicine systems aimed at monitoring diabetes, a recent comprehensive review can be found in [16]. Such a study identifies motivation for selfmanagement, long term adherence, costs, adoption, satisfaction and outcomes as the dimensions to evaluate a PHS. On one hand, with respect to these dimensions we could not evaluate our system from the perspective of costs and clinical outcomes, except for glucose control, as our study implied focused on feasibility of the technology with only 12 patients. On the other hand, the motivations for self-management in GDM are very clear as it is very uncomfortable for pregnant women to visit doctors every week, the adherence happened to be significantly better than with the standard care, there was no dropout rate from the perspective of adoption and the patients and doctors were generally satisfied with the technology, and the system demonstrated an improvement from the perspective of glucose control. With respect to what is suggested by the state-of-the-art on the subject of evaluating telemedicine technology, it is clear that we need to run a bigger trial with considerably more patients to be able to evaluate costs and clinical outcomes.

Furthermore, as reported by the authors of [16], only 9\% of the studies considered in their review focus on decision support techniques as we do in our contribution and of this $9 \%$ none focuses on GDM, and only one focuses on telemedicine applied to GDM. From this perspective, we believe that our agent based PHS to monitor GDM represents and advancement to the state-of-the-art, as it covers an important gap in the area of diabetes monitoring.

A related and parallel work on which the authors are also involved is COMMODITY12 [21]. COMMODITY12, like the system presented in this paper, is also making use of event calculus and GOLEM. The main contributions of this paper with respect fo COMMODITY12 are: a) We performed a trial on real patients, whereas COMMODITY12 is still in prototypical stage; b) We model the knowledge to handle glucose control in GDM, which is not considered in COMMODITY12; c) We model the mobile user interface; d) We provide web interface modelled on the needs of the medical doctors; e) We evaluate the experience of the users; f) The presented system also evaluates the experience of the medical doctors. With respect to the presented system, COMMODITY12 is more advanced from the perspective of interoperability. Interoperability using HL7 is an important problem, but within this contribution we preferred to focus on the acceptability of the technology from the perspective of the patients. In future extensions of the GDM system presented, we will approach the problem of interoperability at the level of the smarthub, but this was out of the scope for the current contribution.

Amongst those PHS offering decision support, our system presents similarities with the works presented in [31], [25], [4] and [5]. In [31], Quinn et al. present WellDoc, a mobile phone based system to provide patients with real time feedback on their glucose levels. WellDoc shows an improvement on the glucose control of the patients as compared to standard care. With respect to WellDoc, our system focuses mainly on providing decision support to the doctors rather than to the patient, although, the interface of our GDM system provides graphs as an immediate feedback to the patients. It was decided in collaboration with medical doctors to limit direct feedback to the patient to avoid to create anxiousness as this may have negative effects on the treatment. The work of Lim et al. in [25] is very close to ours as they use a rule engine to deal with the alerts in the system. Similarly to [31], the system presented in [25] provides advice directly to the patient, whereas we prefer to provide decision support to the caregivers who then are in charge of modifying the treatment of the patient.

Another important approach towards diabetes management is represented by Diabeo [13]: Diabeo implements a bolus calculator to provide advice to dia- 
betic type 1 patients. Diabeo is orthogonal to our work as we do not consider the effects of bolus intake. It could represent a future direction to combine Diabeo and the calculation of energy expenditure to provide decision support on the amount of medicament the patient should take.

The work of Cafazzo et al. [12] presents an interesting approach towards monitoring of diabetes type 1 in young patients. In such a contribution, the focus was on monitoring the glucose control and the collection of glucose values by means of gamification patterns, showing an improvement in the overall treatment management of the patients. With respect to the contribution in [12], our system does not propose gamification patterns as we preferred to keep an approach that was as less invasive in the privacy of the patient as possible. With respect to the contribution of Cafazzo et al. the main novelty of our system is to consider alerts in terms of temporal patterns of the physiological values.

Almost in parallel with the submission of this contribution, Garcia-Saez et al. presented a system similar to ours in [17]. Such a system modelled computer interpretable guidelines to monitor the health status of the patients, her compliance and to inform her about relevant events. With respect to the work of GarciaSaez et al., our main contribution is the enactment of medical knowledge by means of logic programming and agents, which makes our approach flexible as we have a separation between infrastructure and knowledge management problems. Furthermore, the use of the EC formalism, allows us to model the evolution in time of the patient health, which allows us to represent alerts in terms of a repetition of events.

Considering related works from the broader perspective of pervasive health systems to monitor chronic illnesses, an extensive review on the subject can be found in [27]. Given the classification of system flexibility proposed in [27], our system is a multi-function system where we offer the following services: alerting, support activities, information and documentation, analytical and diagnostic support. From the complexity of use perspective, since for the moment we do not consider the use of sensors, we have a single component system. From this review, the works $[1,35]$ are the most related to ours.

The work of Tentori et al. [37] has aspects similar to our model, but the focus of their PHS is rather on applying machine learning techniques, such as Hidden Markov Models (HMM) for activity recognition. Our choice of formalizing medical guidelines rather than using machine learning techniques has been dictated by the needs of our medical doctors: the main issue when dealing with automatic alerts is to be able to interpret the results. As it has been widely discussed, machine learning is more precise than rules when dealing with classification problems, the main issue though is that it is the interpretability of the classification model, consequently the final user loses trust in the application as if a mistake occurs, there is not an easy way to explain it, while with a formal approach, the rule is still readable by an expert. Furthermore, the medical doctor has more trust on the rule due to the fact that this is formalized directly from his experience. In the case of the GDM experts we deployed, the application of temporal rules is also possible because we are dealing with discrete and sparse signals such as the glucose values observed during the day and the week. In the case the signal becomes more dense, the introduction of machine learning models as those deployed by Tentori et al., in addition to the already deployed rules, would certainly be necessary.

In [1] the AMON project is presented. In AMON, patients affected by heart issues are monitored using a mobile solution. In AMON the monitoring is performed with an un-obtrusive device integrating several sensors in one solution. AMON is clearly more advanced than our system for what it concerns the monitoring of multi-parametric physiological values, but it does not propose a formalization of guidelines for decision support as we do. In this sense, the next step for us it to employ a solution similar to AMON to extend the application of guidelines to a multi-parametric solution.

In [35] a rule based solution is applied to data coming from automatic-defibrillators in home care settings. As for our case, the solution demonstrated users' satisfaction for the automatic interpretation of data and the ease of use. Similarly to our results, the survey presented a high acceptability amongst the patients and the doctors. By comparing with [35], the use of a rule based approach to telemonitoring of patients seems to be quite convenient thanks to the understandability of the rules.

\section{Conclusion and Future Work}

In this paper we presented a PHS to monitor patients affected by gestational diabetes. We presented the system functionality, the constraints that we took into consideration when developing the application and the agent cognitive model we developed for monitoring 
patient's physiological values. This paper also present the results of a feasibility study that we have run on 12 patients affected by GDM in the University Hospital of Lausanne. The study shows that the system is successful in improving glycaemic control in the patients and that the system allows the patients to feel more confident in their pregnancy. We also presented a qualitative analysis concerning the satisfaction of the patients and caregivers involved in the study, showing positive results.

As a matter of future work we plan to develop datadriven algorithms on the data collected with our PHS, following an approach similar to the one proposed in [3], for the prediction of hypoglycaemia and hyperglycaemia events, to improve the glucose control in our patients.

The implementation of interoperability standards within the GDM system is going to be important future work to ensure the further acceptability of the technology within caregivers environment, similarly to the work we performed in [21].

Similarly, we plan to use the collected data to define treatment adjustment rules for the doctors, to provide further decision support in the treatment of the patients affected by GDM.

Another possible direction concerns the inclusion of further rules to deal with onset of preeclampsia and macrosomia in those patients whose glucose control is poor. In this sense, a natural further step for our system would be to consider continuous glucose sensors to analyze patterns in the patient's behavior and thus provide prediction facilities to the caregivers.

The graphs and charts provided in this study were of simple nature, we think that more elaborated charts and a representation of the data that puts physiological patterns in relation may allow the medical doctors to have a better understanding of the health of the patient, thus improving their ability to effectively modify the treatment plan. Similarly, in the current presentation of the alerts, we only highlight patterns getting out of the normal threshold. We think that an interesting development could be to highlight the repetition of alarming patterns so that the medical doctors and the patients are notified of these cyclical events in order to tackle the reason behind these patterns.

Finally, we also envisage to extend the sensor base and use further non invasive sensors for the monitoring of other physiological values of the patients, such as blood pressure and weight.

\section{Acknowledgment}

This work was partially funded by the Hasler Stiftung and by the Nano-Tera grant 10020 . This work was partially supported by the FP7 287841 COMMODITY12 project.

\section{References}

[1] Urs Anliker, Jamie A. Ward, Paul Lukowicz, Gerhard Tröster, François Dolveck, Michel Baer, Fatou Keita, Eran B. Schenker, Fabrizio Catarsi, Luca Coluccini, Andrea Belardinelli, Dror Shklarski, Menachem Alon, Etienne Hirt, Rolf Schmid, and Milica Vuskovic. Amon: a wearable multiparameter medical monitoring and alert system. IEEE Transactions on Information Technology in Biomedicine, 8(4):415-427, 2004.

[2] Alexander Artikis, Marek Sergot, and Georgios Paliouras. A logic programming approach to activity recognition. In Proceedings of the 2nd ACM international workshop on Events in multimedia, EiMM '10, pages 3-8, New York, NY, USA, 2010. ACM.

[3] R. Bellazzi and A. Abu-Hanna. Data mining technologies for blood glucose and diabetes management. J Diabetes Sci Technol, 3(3):603-612, May 2009.

[4] R. Bellazzi, C. Larizza, S. Montani, A. Riva, M. Stefanelli, G. d'Annunzio, R. Lorini, E. J. Gomez, E. Hernando, E. Brugues, J. Cermeno, R. Corcoy, A. de Leiva, C. Cobelli, G. Nucci, S. Del Prato, A. Maran, E. Kilkki, and J. Tuominen. A telemedicine support for diabetes management: the T-IDDM project. Comput Methods Programs Biomed, 69(2):147-161, Aug 2002.

[5] Y. Boukhors, R. Rabasa-Lhoret, H. Langelier, M. Soultan, A. Lacroix, and J. L. Chiasson. The use of information technology for the management of intensive insulin therapy in type 1 diabetes mellitus. Diabetes Metab., 29(6):619-627, Dec 2003.

[6] M. E. Boyd, R. H. Usher, and F. H. McLean. Fetal macrosomia: prediction, risks, proposed management. Obstet Gynecol, 61:715-722, Jun 1983.

[7] Stefano Bromuri, Michael Schumacher, Kostas Stathis, and Juan Ruiz. Monitoring Gestational Diabetes Mellitus with Cognitive Agents and Agent Environments. In Proceedings of the 2011th IEEE/WIC/ACM International Conference on Intelligent Agent Technology (IAT 2011), Aug 2011.

[8] Stefano Bromuri, Michael Ignaz Schumacher, and Kostas Stathis. Towards Distributed Agent Environments for Pervasive Healthcare. In Jürgen Dix and Cees Witteveen, editors, MATES, volume 6251 of Lecture Notes in Computer Science, pages 125-137. Springer, 2010.

[9] Stefano Bromuri, Michael Ignaz Schumacher, and Kostas Stathis. A self-healing distributed pervasive health system. Int. J. Web Eng. Technol., 8(2):177-196, 2013.

[10] Stefano Bromuri and Kostas Stathis. Distributed Agent Environments in the Ambient Event Calculus. In DEBS '09: Proceedings of the third international conference on Distributed event-based systems, New York, NY, USA, 2009. ACM.

[11] Stefano Bromuri, Visara Urovi, and Kostas Stathis. iCampus: A Connected Campus in the Ambient Event Calculus. International Journal of Ambient Computing and Intelligence, 2(1):59-65, 2010. 
[12] A. Joseph Cafazzo, Mark Casselman, Nathaniel Hamming, K. Debra Katzman, and R. Mark Palmert. Design of an mhealth app for the self-management of adolescent type 1 diabetes: A pilot study. J Med Internet Res, 14(3):e70, May 2012.

[13] Guillaume Charpentier, Pierre-Yves Benhamou, Dured Dardari, Annie Clergeot, Sylvia Franc, Pauline SchaepelynckBelicar, Bogdan Catargi, Vincent Melki, Lucy Chaillous, Anne Farret, Jean-Luc Bosson, Alfred Penfornis, and on behalf of the TeleDiab Study Group. The Diabeo software enabling individualized insulin dose adjustments combined with telemedicine support improves hbalc in poorly controlled type 1 diabetic patients: A 6-month, randomized, open-label, parallelgroup, multicenter trial (TeleDiab 1 study). Diabetes Care, 34(3):533-539, 2011.

[14] L. Chittaro, A. Montanari, M. Dojat, and C. Gasparini. The Event Calculus at work: a case study in the medical domain. In Intelligent Systems Engineering, 1994., Second International Conference on Intelligent Systems Engineering, pages 195200. IEE Press, Sep 1994.

[15] M. G. Dalfra, A. Nicolucci, A. Lapolla, A. Di Benedetto, G. Di Cianni, M. A. Dolci, I. Franzetti, A. Galluzzo, A. Napoli, G. Salietti, C. Santini, E. Torlone, C. Tortul, and E. Vitacolonna. The effect of telemedicine on outcome and quality of life in pregnant women with diabetes. J Telemed Telecare, 15(5):238-242, 2009.

[16] Omar F. El-Gayar, Prem Timsina, Nevine Nawar, and Wael Eid. A systematic review of IT for diabetes self-management: Are we there yet? I. J. Medical Informatics, 82(8):637-652, 2013.

[17] G. Garcia-Saez, M. Rigla, I. Martinez-Sarriegui, E. Shalom, M. Peleg, T. Broens, B. Pons, E. Caballero-Ruiz, E. J. Gomez, and M. E. Hernando. Patient-oriented Computerized Clinical Guidelines for Mobile Decision Support in Gestational Diabetes. J Diabetes Sci Technol, 8(2):238-246, Mar 2014.

[18] A. S. Grustam, J. L. Severens, J. van Nijnatten, R. Koymans, and H. J. Vrijhoef. COST-EFFECTIVENESS OF TELEHEALTH INTERVENTIONS FOR CHRONIC HEART FAILURE PATIENTS: A LITERATURE REVIEW. Int J Technol Assess Health Care, pages 1-10, Feb 2014.

[19] Peter Hammond and Marek Sergot. Computer support for protocol-based treatment of cancer. The Journal of Logic Programming, 26(2):93 - 111, 1996.

[20] G. Hripcsak. Writing Arden Syntax Medical Logic Modules. Comput. Biol. Med., 24(5):331-363, Sep 1994.

[21] Özgür Kafali, Stefano Bromuri, Michal Sindlar, Tom van der Weide, Eduardo Aguilar-Pelaez, Ulrich Schaechtle, Bruno Alves, Damien Zufferey, Esther RodríguezVillegas, Michael Ignaz Schumacher, and Kostas Stathis. Commodity $_{12}$ : A smart e-health environment for diabetes management. JAISE, 5(5):479-502, 2013.

[22] Catherine Kim, Katherine M. Newton, and Robert H. Knopp. Gestational diabetes and the incidence of type 2 diabetes: a systematic review. Diabetes Care, 25(10):1862-1868, 2002.

[23] R Kowalski and M Sergot. A logic-based calculus of events New Gen. Comput., 4(1):67-95, 1986.

[24] Johannes Krampf, Stefano Bromuri, Michael Schumacher, and Juan Ruiz. An Agent Based Pervasive Healthcare System: a First Scalability Study. In Proceedings of the 4th ICST International Conference on eHealth (eHealth 2011), ICST Lecture Notes (LNICST). Springer Verlag, November 2011.
[25] Soo Lim, Seon Mee Kang, Hayley Shin, Hak Jong Lee, Ji Won Yoon, Sung Hoon Yu, So-Youn Kim, Soo Young Yoo, Hye Seung Jung, Kyong Soo Park, Jun Oh Ryu, and Hak C. Jang. Improved glycemic control without hypoglycemia in elderly diabetic patients using the ubiquitous healthcare service, a new medical information system. Diabetes Care, 34(2):308313, 2011.

[26] D. S. Mastrogiannis, E. Igwe, and C. J. Homko. The role of telemedicine in the management of the pregnancy complicated by diabetes. Curr. Diab. Rep., 13(1):1-5, Feb 2013.

[27] Carsten Orwat, Andreas Graefe, and Timm Faulwasser. Towards pervasive computing in health care - A literature review. BMC Medical Informatics and Decision Making, 8(1):1âĂŞ18, 2008.

[28] E. I. Papageorgiou. Fuzzy cognitive map software tool for treatment management of uncomplicated urinary tract infection. Comput Methods Programs Biomed, 105(3):233-245, Mar 2012.

[29] G. Pare, M. Jaana, and C. Sicotte. Systematic Review of Home Telemonitoring for Chronic Diseases: the Evidence Base. J Am Med Inform Assoc, 14(3):269-277, 2007.

[30] P. Petit, M. Top, F. Chantraine, J. F. Brichant, P. Y. Dewandre, and J. M. Foidart. Treatment of severe preeclampsia: until when and for what risks/benefits? Rev Med Liege, 64:620-625, Dec 2009.

[31] C. C. Quinn, S. S. Clough, J. M. Minor, D. Lender, M. C. Okafor, and A. Gruber-Baldini. WellDoc mobile diabetes management randomized controlled trial: Change in clinical and behavioral outcomes and patient and physician satisfaction. Diabetes Technology and Therapeutics, 10(3):160-168, 2008.

[32] René Schumann, Stefano Bromuri, Johannes Krampf, and Michael Ignaz Schumacher. Agent based monitoring of gestational diabetes mellitus (demonstration). In Wiebe van der Hoek, Lin Padgham, Vincent Conitzer, and Michael Winikoff, editors, AAMAS, pages 1487-1488. IFAAMAS, 2012.

[33] David C. Serlin and Robert W. Lash. Diagnosis and Management of Gestational Diabetes Mellitus. American Family Physician, 80(1):57-62, 2009.

[34] T. Sheeran, T. Rabinowitz, J. Lotterman, C. F. Reilly, S. Brown, P. Donehower, E. Ellsworth, J. L. Amour, and M. L. Bruce. Feasibility and Impact of Telemonitor-based Depression Care Management for Geriatric Homecare Patients. Telemed J E Health, 17(8):620-626, Oct 2011.

[35] A. M. Sinha, R. Koos, K. U. Markus, B. H. Diem, A. Urbaszek, P. Hanrath, and C. Stellbrink. Multicentre evaluation of a rulebased data filter for home monitoring of implanted cardioverter defibrillators. J Telemed Telecare, 12(2):97-102, 2006.

[36] E. G. Spanakis, F. Chiarugi, A. Kouroubali, S. Spat, P. Beck, S. Asanin, P. Rosengren, T. Gergely, and J. Thestrup. Diabetes Management Using Modern Information and Communication Technologies and New Care Models. Interact J Med Res, 1(2):e8, 2012.

[37] Monica Tentori, Marcela Rodriguez, and Jesus Favela. An Agent-Based Middleware for the Design of Activity-Aware Applications. IEEE Intelligent Systems, 26(3):15âĂŞ23, 2011.

[38] Upkar Varshney. Pervasive Healthcare Computing: EMR/EHR, Wireless and Health Monitoring. Springer Publishing Company, Incorporated, 2009.

[39] J. M. Warren. Pregnancy outcomes in women with gestational diabetes compared with the general obstetric population. $\mathrm{Ob}$ stet Gynecol, 91:638-639, Apr 1998. 
S. Bromuri et al. / An Expert Personal Health System to Monitor Patients Affected by Gestational Diabetes Mellitus: A Feasibility Study 19

[40] J. M. Warren. Pregnancy outcomes in women with gestational diabetes compared with the general obstetric population. $\mathrm{Ob}$ stet Gynecol, 91:638-39, Apr 1998.

[41] M. Wooldridge. MultiAgent Systems. John Wiley and Sons, 2002 .
[42] Wendy Van Wootten and R. Elaine Turner. Macrosomia in neonates of mothers with gestational diabetes is associated with body mass index and previous gestational diabetes. Journal of the American Dietetic Association, 14(1):5-30, 2002. 